\title{
Antioxidants: Fascinating and Favourable Biomolecules for Humans
}

\author{
Chandani T. Desai ${ }^{1, ~ *, ~ G a u r a v ~ S . ~ S h a h ~}{ }^{2}$ \\ ${ }^{1}$ Biotechnology Department, Shree Ramkrishna Institute of Computer Education and Applied Sciences, Surat, Gujarat, India \\ ${ }^{2}$ Biotechnology Department, Veer Narmad South Gujarat University, Surat, Gujarat, India \\ Email address: \\ chandani.desai06@gmail.com (C. T. Desai),drgaurav@vnsgu.ac.in (G. S. Shah) \\ To cite this article: \\ Chandani T. Desai, Gaurav S. Shah. Antioxidants: Fascinating and Favourable Biomolecules for Humans. Science Innovation. \\ Vol. 3, No. 6, 2015, pp. 113-116. doi: 10.11648/j.si.20150306.18
}

\begin{abstract}
Free radicals are highly reactive molecules or chemical species that are competent enough of independent existence. Free radicals can cause "oxidative stress," a process that can trigger cell damage. Oxidative stress is thought to play a role in a variety of diseases including cancer, cardiovascular diseases, diabetes, Alzheimer's disease, Parkinson's disease. Increasing the antioxidant intake can prevent diseases and lower the health problems. Generation of highly Reactive Oxygen Species (ROS) is an integral feature of normal cellular function like mitochondrial respiratory chain, phagocytosis, arachidonic acid metabolism, ovulation, and fertilisation. Antioxidants combat oxidative stress by working to neutralise excess free radicals and stopping them from starting the chain reactions that contribute to various diseases and early aging. Different types of natural antioxidants are present in fruit and vegetables; they have synergistic interactions that are important due to their activity and regenerative potential.
\end{abstract}

Keywords: Antioxidants, Oxidative Stress, Reactive Oxygen Species, Epigallocatechin Gallate

\section{Introduction}

Free radicals are highly unstable molecules that are naturally formed when you exercise and when your body converts food into energy. Your body can also be exposed to free radicals from a variety of environmental sources, such as cigarette smoke, air pollution and sunlight. Research is increasingly showing that antioxidant rich foods, herbs bring in health benefits. Foods may possibly enhance antioxidant levels because foods contain a lot of antioxidant substances. Fruits and vegetables are loaded with key antioxidants such as vitamin $\mathrm{A}, \mathrm{C}, \mathrm{E}, \boldsymbol{\beta}$ - carotene and important minerals, including selenium and zinc. Fruits, vegetables and medicinal herbs are the richest sources of antioxidant compounds [Sies H et al, 1992]. Additionally, vitamin $\mathrm{E}$ also possesses antiproliferative properties that interfere in signal transduction and in inducing cell cycle arrest.

By the late 1950s, it was shown that oxidation reactions are involved in aging and the progression of several diseases and it was proposed that antioxidant molecules may slow down the aging process, disease progression and prolong the lifespan [Gutteridge, J.M.C.; Halliwell, B,
2010.]. Antioxidants are substances that, at low concentrations, prevent or retard the oxidation of easily oxidisable biomolecules such as lipids, proteins and DNA [Becker, E.M et al, 2004]. Earlier, antioxidants were simply defined; as substances which counteract free radicals, thus preventing oxidative damage. [Ratnam, D.V et al, 2006]. Two major groups of antioxidants are recognised, namely enzymatic and non - enzymatic antioxidants. Enzymatic antioxidants include the primary enzymes, superoxide dismutase, catalase and glutathione peroxidase, the secondary enzymes, glutathione reductase and glucose - 6 phosphate dehydrogenase [Ratnam, D.V et al, 2006]. Non enzymatic antioxidants, are either water - soluble (vitamin $\mathrm{C}$ and phenolic compounds) or lipid - soluble (vitamin E and carotenoids) biomolecules [Podsędek, A, 2007]. In humans the over - production of Reactive Oxygen Species (ROS) can result in tissue injury and has been implicated in disease progression and oxidative damage of nucleic acids and proteins [Middleton, Jr. E, 2008]. When there is a lack of antioxidants to quench the excess reactive free radicals, cardiovascular, cancer, neurodegenerative, Alzheimer's and inflammatory diseases may develop in the body [Krishnaiah, D et al, 2010]. 


\section{Antioxidants in Noise Induced Hearing Loss}

The role of antioxidants in attenuating hearing loss has been the subject of much interest, scrutiny, criticism and controversy over the past several years. Presbyacusis is the progressive, high frequency, sensorineural hearing loss that occurs with increasing age. The accumulation of ROS is strongly implicated in the aging process and is regarded as an important deleterious event in the genesis of presbyacusis [Head E et al, 2002]. The effects of an antioxidant - rich diet in beagle dogs on age - related cochlear structural changes was studied and it was found that the dogs that were fed a diet rich in vitamin E, L-carnitine, DL-a-lipoic acid, and vitamin $\mathrm{C}$ in the last 3 years of their lives exhibited a greater neuronal density in the apical turn of the spiral ganglion versus an age - matched control group [Le T, Keithley EM, 2007].

Noise - induced hearing loss (NIHL) is a type of high frequency sensorineural hearing loss, often with a classic notch at $4000 \mathrm{~Hz}$. It is a preventable cause of hearing loss caused by excessive exposure to either acute or chronic acoustic trauma. Research [Le Prell CG et al, 2007] has been aimed at investigating antioxidants to treat or prevent the onset or progression of NIHL. It has been observed that, with massive noise stimulation, an excessive amount of glutamate, a neurotransmitter at the junction of the inner hair cells and afferent neuron in the peripheral auditory system, is released resulting in ionic influx and massive entry of water and extracellular calcium into the afferent neurons, causing cell death and noise induced hearing loss [Duan M et al, 2009; Spoendlin H.1971]. The protective effect of caroverine, an antioxidant and an antagonist of two glutamate receptors, has been studied earlier against acoustic trauma in rats. The study revealed that prior to impulse noise insult, subcutaneously administered caroverine decreases the impulse noise induced hearing loss in rats. [Duan $\mathrm{M}$ et al, 2006]

\section{Antioxidants in Neurodegenerative Disorders}

Neurodegenerative disorders are a heterogeneous group of diseases of the nervous system, including the brain, spinal cord and peripheral nerves, that have many different aetiologies. Many are hereditary, some are secondary to toxic or metabolic processes and others result from infections. The production of free radicals, multiplies several folds during pathological conditions. The release of oxygen free radicals has also been reported during the recovery phases from many pathological noxious stimuli to the cerebral tissues [Halliwell B, Gutteridge JMC, 1989]. The high lipid content of nervous tissue, coupled with its high aerobic metabolic activity, makes it particularly susceptible to oxidative damage. The high level of iron may be essential, particularly during brain development, but its presence also means that injury to brain cells may release iron ions, which lead to oxidative stress via the iron - catalysed formation of ROS [Bauer V, Bauer F, 1999; Andorn AC et al, 1990]. A number of in vitro studies have shown that antioxidants both endogenous and dietary can protect nervous tissue from damage by oxidative stress [Contestabile A., 2001]. Uric acid which is an endogenous antioxidant was found to prevent neuronal damage in rats, both in vitro and in vivo, from the metabolic stresses of ischaemia, oxidative stress as well as exposure to the excitatory amino acid glutamate. The most widely studied dietary antioxidants are vitamin $\mathrm{C}$, vitamin $\mathrm{E}$ and beta carotene. Vitamin $\mathrm{C}$ is considered the most important water soluble antioxidant in extracellular fluids, as it is capable of neutralising ROS in the aqueous phase before lipid peroxidation is initiated. Vitamin E is a major lipid - soluble antioxidant and is the most effective chain-breaking antioxidant within the cell membrane where it protects membrane fatty acids from lipid peroxidation. $\beta$ - carotene and other carotenoids also provide antioxidant protection to lipid rich tissues. Fruits and vegetables are major sources of vitamin $\mathrm{C}$ and carotenoids, while whole grains, i.e., cereals and high quality vegetable oils are major sources of vitamin E [Halliwell B, 1994; Jacob RA, 1995; Block G et al, 1992]. It has been studied that vitamin $\mathrm{E}$ prevents cell death (apoptosis) in rat neurons subjected to hypoxia followed by oxygen reperfusion [Yu ZF et al, 1998]. In the same study it was shown that vitamin $E$ prevented neuronal damage from reactive nitrogen species. Both vitamin $\mathrm{E}$ and $\beta$-carotene were found to protect rat neurons against oxidative stress from exposure to ethanol (Copp RP et al, 1999). In an experimental model of diabetes - caused neurovascular dysfunction, $\beta$-carotene was found to protect cells most effectively, followed by vitamin $\mathrm{E}$ and vitamin $\mathrm{C}$ (Mitchell JJ et al, 1999).

\section{Antioxidants in Cancer}

Antioxidants are capable of donating electrons to stabilize ROS and to inhibit their detrimental effects, including both endogenous (synthesized by the body itself) and exogenous molecules (those from external sources to the body) [Uttara, $\mathrm{B}$ et al, 2009]. Oxidative Stress (OS) is involved in the process of the development of cancer and tumors, due to that ROS can damage the macromolecules as lipids, which react with metals (such as free iron and copper) and produce aldehydes and synthesize malondialdehyde - inducing mutations [Noda, N.\&Wakasugi, H. 2000] or cause breaks in the double chain, produce modifications in guanine and thymine bases and sister chromatid exchanges [Brown, N. S., \& Bicknell, R. 2001].Oxidative damage or genetic defects that result in some defective enzymes that are incapable of repairing the mutations increase the incidence of age dependent cancer [Halliwell, B. 2007]. It has been proposed that lower antioxidant activity increases the risk of developing cancer; thus, ingestion of antioxidants can prevent cancerogenesis. Antioxidant phenolic agents have been implicated in the mechanisms of chemoprevention, which refers to the use of chemical substances of natural or 
of synthetic origin to reverse, retard or delay the multistage carcinogenic process [Ebenezer, O et al, 2011]. Dietary polyphenols may exert their anticancer effects through several possible mechanisms, such as removal of carcinogenic agents, modulation of cancer cell signaling and antioxidant enzymatic activities and induction of apoptosis as well as of cell cycle arrest. Some of these effects may be related, at least partly, with their antioxidant activities $[\mathrm{Hu}$, M.L. 2011]. They may exert protective effects against cancer development, particularly in the gastrointestinal tract, where they will be at their highest concentration. In fact, many studies have shown that various polyphenol - rich fruits and vegetables are particularly effective in protecting against colon cancer development [Martinez, M. E. 2005; Li, Q et al, 2009]. Many of the anti - cancer properties associated with green tea are thought to be mediated by the flavanol Epigallocatechin gallate (EGCG), which has been shown to induce apoptosis and inhibit cancer cell growth by altering the expression of cell cycle regulatory proteins and the activity of signalling proteins involved in cell proliferation, transformation and metastasis [Khan, $\mathrm{N}$ et al, 2006]. In-vivo studies have demonstrated that many natural compounds found in plants and fruits have the capability to inhibit many types of human and animal cancer. Vitamins such as C, E and A have shown that they can diminish cervical, bladder, prostate, intestinal, skin and other gastrointestinal cancer types and that they have the capability to inhibit ROS production in patients [Fuchs - Tarlovsky et al, 2011; Fukumura, H et al, 2012; Mazdak, H., \& Zia, H. 2012; Thapa, D., \& Ghosh, R. 2012; Szpetnar, M et al, 2012; Jayaprakash, V., \& Marshall, J. R. 2011; Slaga, T. J. 1995].

\section{Conclusion}

Oxidants and free radicals are inevitably produced during the majority of physiological and metabolic processes and the human body has defensive antioxidant mechanisms; these mechanisms vary according to cell and tissue type and may act antagonistically or synergistically. Enzymatic antioxidant defenses include Superoxide dismutase (SOD), Glutathione peroxidise (GPx), and Catalase (CAT). Non - enzymatic antioxidants are represented by ascorbic acid (vitamin C), $\alpha$ tocopherol (vitamin E), Glutathione (GSH), carotenoids, flavonoids, tannins, triterpepenoids, saponins, glycosides, steroids and other antioxidants [Gupta, V, \& Sharma, M. 2012] Under normal conditions, there is a balance between both the activities and the intracellular levels of these antioxidants: this equilibrium is essential for the survival of organisms and their health. However, it has been observed earlier about the ascribed health effects of antioxidants in many studies that are mainly based on in - vitro assays which do not necessarily reflect the human physiological mechanisms in - vivo. These controversies on the effectiveness of antioxidants have stimulated research on in - vivo studies relating to their bioavailability to bridge the gap with in-vitro observations [Becker et al, 2004; Shen et al, 2007].

\section{References}

[1] Andorn AC, Britton RS, Bacon BR. (1990) Evidence that lipid peroxidation and total iron are increased in Alzheimer's brain. Neurobiol Aging 11: 316.

[2] Bauer V, Bauer F. (1999) Reactive oxygen species as mediators of tissue protection and injury. Gen PhysiolBiophys 18: 7 - 14.

[3] Becker, E. M.; Nissen, L. R.; Skibsted, L. H. (2004) Antioxidant evaluation protocols: Food quality or health effects. Eur. Food Res. Technol219: 561 - 571.

[4] Block G, Patterson B, Subar A. (1992) Fruits, vegetables and cancer prevention: A review of epidemiological evidence. NutrCancer 18: 1 - 29.

[5] Brown, N. S., \& Bicknell, R. (2001). Hypoxia and oxidative stress in breast cancer: Oxidative stress: its effects on the growth, metastatic potential and response to therapyof breast cancer. Breast Cancer Research, 3: 323 - 327, 0167 - 6806, Print, 1573 - 7217, (Online).

[6] Contestabile A. (2001) Oxidative stresses in neurodegeneration: mechanisms and therapeutic perspectives. Curr Top MedChem1 (6): 553 - 68.

[7] Copp RP, Wisniewski T, Hentatin F et al. (1999) Localisation of alphatocopherol transfer protein in the brains of patients with ataxia with vitamin E deficiency and other oxidative stress related neurodegenerative disorders. Brain Res822 (1 2): $80-7$.

[8] Duan M, Chen Z, Qiu J, et al. (2006) Low - dose, long - term caroverine administrationattenuates impulse noise - induced hearing loss in the rat. ActaOtolaryngologica.This paper investigates the use of low - dose, long - term caroverine as a novel drug to protect against NIHL. 126: 1140-1147.

[9] Duan M, Agerman K, Ernfors, Canlon B. (2009) Complementary roles of neurotrophin 3 and an $\mathrm{N}$ - methyl - D - aspartate antagonist in the protection of noise and aminoglycoside - induced ototoxicity. Pro Natl AcadSci U S A97: 7597-7602.

[10] Ebenezer, O., Farombi, A., \&Olatunde. (2011). Antioxidative and chemopreventiveproperties of Vernoniaamygdalina and Garcinia biflavonoid. International Juornal of Environment Researc and Public Health, 8: 2533 - 2555, 1661 - 7827, Print, 1660 - 4601, (Online).

[11] Fuchs - Tarlovsky, V., Bejarano - Rosales, M., Gutierrez Salmeán, G., Casillas, MA, López - Fukumura, H, Sato, M, Kezuka, K, Sato, I, Feng, X, Okumura, S, Fujita, T, Yokoyama, U, Eguchi, H, Ishikawa, Y, \& Saito, T. (2012). Effect of ascorbic acid on reactive oxygen species production in chemotherapy and hyperthermia in prostate cancer cells.The Jornal of Physiological Sciences, 1880 - 6546, (Print), 1880 6562, Online, 62: 251 - 257.

[12] Fukumura, H, Sato, M, Kezuka, K, Sato, I, Feng, X, Okumura, S, Fujita, T, Yokoyama, U, Eguchi, H, Ishikawa, Y, \& Saito, T. (2012). Effect of ascorbic acid on reactive oxygenspecies production in chemotherapy and hyperthermia in prostate cancer cells. The Jornal of Physiological Sciences, 1880 6546, (Print), 1880 - 6562, Online, 62: 251 - 257. 
[13] Gupta, V, \& Sharma, M. (2012). Phytochemical Analysis and Evaluation of AntioxidantActivities of Methanolic Extracts of Maytenusemarginata. 1536 - 2310, (Print), 1557 - 8100, Online, 16 (5): 257 - 262.

[14] Gutteridge, J. M. C.; Halliwell, B. (2010). Antioxidants: Molecules, medicines and myths. Biochem. Biophys. Res. Commun., 393: 561 - 564.

[15] Halliwell B, Gutteridge JMC. (1989) In free radicals in Biology and Medicine, 2nd Ed, Oxford University Press, Oxford, UK.

[16] Halliwell, B. (2007). Oxidative stress and cancer: have we moved forward? BiochemicalJournal, 401: 1 - 11, 0264 6021, Print, 1470 - 8728, (Online).

[17] Halliwell B. (1994) Free radicals, antioxidants and human disease: curiosity, cause or consequences? Lancet, 344: 721 4.

[18] Head E, Liu J, Hagen TM, et al (2002). Oxidative damage increases with age in a canine model of human brain aging. JNeurochem82: 375-381.

[19] Hu, M. L. (2011). Dietary Polyphenols as Antioxidants and Anticancer Agents: MoreQuestions than Answers. Chang Gung Medical Journal, 2072 - 0939, 34: 449 - 459.

[20] Jacob RA (1995). The integrated antioxidant system. Nutr Res 15 (5): 755 - 66.

[21] Jayaprakash, V., \& Marshall, J. R. (2011). Selenium and other antioxidants for chemoprevention of gastrointestinal cancers. Best Practtice\& Research Clinical Gastroenterology, 25: 507 - 518, $1521-6918$.

[22] Khan, N., Afaq, F., Saleem, M., Ahmad, N., \& Mukhtar, H. (2006). Targeting multiplesignaling pathways by green tea polyphenol (-) - epigallocatechin - 3 - gallate. Cancer Research, 66: 2500 - 2505, 0008 - 5472, Print, 1538 - 7445, Online.

[23] Krishnaiah, D.; Sarbatly, R.; Nithyanandam, R. (2010) A review of the antioxidant potential of medicinal plant species. Food Bioprod. Process., doi: 10.1016/j.fbp.2010.04.008.

[24] Le Prell CG, Yamashita D, Minami SB, et al. (2007). Mechanisms of noise - induced hearing loss indicate multiple methods of prevention. Hear Res. This is an excellent comprehensive review of the mechanism of NIHL and antioxidant effects on NIHL226: 22-43.

[25] Le T, Keithley EM. (2007). Effects of antioxidants on the aging inner ear. This paper illustrates the importance of an antioxidant rich diet on the auditory system. Hear Res226: 194-202.

[26] Li, Q., Zhao, H. F., Zhang, Z. F., Liu, Z. G., Pei, X. R., Wang, J. B., Cai, M. Y., \& Li, Y. (2009). Long - term administration of green tea catechins prevents age - related spatiallearning and memory decline in $\mathrm{C} 57 \mathrm{BL} / 6 \mathrm{~J}$ mice by regulating hippocampal cyclicAMP - response element binding protein signaling cascade. Neuroscience, 159: 1208 - 1215, 03064522 .

[27] Martinez, M. E. (2005). Primary prevention of colorectal cancer: Lifestyle, nutrition, exercise. Recent Results in Cancer Research, 166: 177 - 211, 0080 - 0015.
[28] Mazdak, H., \& Zia, H. (2012). Vitamin e reduces superficial bladder cancer recurrence: a randomized controlled trial. International Journal of Preventive Medicine, 3: 110 - 115.

[29] Mitchell JJ, Paiva M, Heaton MB. (1999). Vitamin E and beta - carotene protect against ethanol combined with ischaemia in an embryonic rat hippocampal culture model of fetal alcohol syndrome. Neurosci Lett 263 (2 - 3): 189 - 92.

[30] Middleton, Jr. E.; Kandaswami, C.; Theoharides, T.C. (2008). The effects of plant flavonoids on mammalian cells: Implication for inflammations, heart disease and cancer. Pharmacol. Rev. 52: 673 - 751.

[31] Noda, N., \&Wakasugi, H. (2000). Cancer and oxidative stress. Journal of the JapanMedical Association, 124 (11): 1571 $1574,1356-8650$.

[32] Podsędek, A. (2007) Natural antioxidants and antioxidant activity of Brassica vegetables: A review. LWT - Food Sci. Technol., 40: 1 - 11.

[33] Ratnam, D. V.; Ankola, D. D.; Bhardwaj, V.; Sahana, D. K.; Kumar, M.N.V.R. (2006).Role of antioxidants in prophylaxis and therapy: A pharmeumaceutical perspective. J. Control Release.113: 189 - 207.

[34] Shen, L.; Hong - Fang, J.; Hong - Yu, Z. (2007) How to understand the dichotomy of antioxidants. Biochem. Biophys. Res. Commun. 362: 543 - 545.

[35] Sies H, Stahl W, Sundquist AR, (1992)Antioxidant function of vitamins, vitamins $\mathrm{E}$ and $\mathrm{C}$, beta - carotene, and other carotenoids, Annals of the New York Academy of Science, 669: 7 - 20 .

[36] Slaga, T. J. (1995). Inhibition of the induction of cancer by antioxidants. Advances inExperimental Medicine and Biology, 369: 167 - 174, 0065 - 2598.

[37] Spoendlin H. (1971) Primary structural changes in the organ of corti after acoustic overstimulation. ActaOtolaryngol (Stockh) 71: 166-176.

[38] Szpetnar, M., Matras, P., Kiełczykowsk, a, M., Horecka, A., Bartoszewska, L., Pasternak, K., \&Rudzki, S. (2012). Antioxidants in patients receiving total parenteral nutritionafter gastrointestinal cancer surgery. Cell Biochemistry and Funciont, 30: 211 - 216, 1099 - 0844, Online.

[39] Thapa, D., \& Ghosh, R. (2012). Antioxidants for prostate cancer chemoprevention: Challenges and opportunities. Biochemical Pharmacology, 83: 1319 - 1330, 0006 - 2952.

[40] Uttara, B., Singh, A. V., Zamboni, P., \& Mahajan, R. T. (2009) Oxidative Stress and Neurodegenerative Diseases: A Review of Upstream and Downstream AntioxidantTherapeutic Options. Current Neuropharmacology. 7: 65 - 74, 0157 - 0159 $\mathrm{X}$.

[41] Yu ZF, Bruce - Keller AJ, Goodman Y, Mattson, MP. (1998) Uric acid protects neurons against excitotoxic and metabolic insults in cell culture, and against focal ischaemic brain injury invivo. J Neurosci Res. 53 (5): 613 - 25. 